\title{
Antigenic heterogeneity in patients with reactions in borderline leprosy
}

\author{
R STC BARNETSON， G BJUNE，J M H PEARSON， G KRONVALL
}

British Medical fournal, 1975, 4, 435-437

\begin{abstract}
Summary
Fifteen patients with borderline leprosy who developed "reversal" reactions were studied from the inception of treatment. Thirteen showed an appreciable increase in lymphocyte transformation (LT) when preparations of Mycobacterium leprae were used as antigen. The LT responses to either "whole" or "sonicated" preparations of the bacillus in these 15 patients and in nine others also in reaction correlated with the clinical presentation. Those with skin disease predominating in the reaction showed an appreciable increase in LT when whole $M$ leprae was used as antigen. Those with nerve disease predominating showed an increase with sonicated $M$ leprae. In those with both skin and nerve disease there was an increase with both antigen preparations. The ratios of the LT test results (whole to sonicated M leprae) showed highly significant differences between the three groups.
\end{abstract}

\section{Introduction}

Mycobacterium leprae, the causative organism of leprosy, is an obligate intracellular parasite with an affinity for skin and nerve. It is remarkably non-toxic-patients may have $10^{12}-10^{13}$ bacilli in the body without systemic illness - and most of the clinical manifestations of the disease result from the host response. The most important cause of deformity in leprosy is nerve damage due to hypersensitivity to bacilli lying in the nerves. Such damage may result from two different mechanisms, both termed "reaction."

"Reversal" reactions, which occur in borderline leprosy, are probably due to a delayed hypersensitivity reaction (Coombs and Gell type IV hypersensitivity reaction), as there is usually a dense lymphocytic infiltration in histological specimens of skin and nerve and an appreciable increase in lymphocyte transformation (LT) when "whole" $M$ leprae is used as antigen.'

"Erytherna nodosum leprosum," which occurs in lepromatous leprosy and is associated with immune complex formation, ${ }^{2}$ is an example of a Coombs and Gell type III hypersensitivity reaction. Nerve damage rarely results from this type of reaction unless it recurs frequently.

Patients with reversal reactions may vary considerably in their clinical presentation and may be divided into three groups-(1) those with skin hypersensitivity predominating, in which there is erythema and oedema of the skin lesions without obvious disease of the nerves; (2) those with nerve hypersensitivity

\footnotetext{
Medical Research Council Leprosy Project, PO Box 1005, Addis Ababa, Ethiopia

R STC BARNETSON, MRCP, clinical research physician

J M H PEARSON, MRCP, clinical research physician

Armauer Hansen Research Institute, PO Box 1005, Addis Ababa, Ethiopia

G BJUNE, MD, immunologist

G KRONVALL, MD, director of institute
}

predominating, in which there is swelling and tenderness of the nerves but no change in the hypopigmented skin lesions; and (3) those with both skin and nerve hypersensitivity.

We describe here 24 patients who developed reversal reactions.

\section{Patients and methods}

Fifteen patients were included in a prospective study of the immunology of borderline leprosy; pretreatment assessments (including LT responses) were available. Nine further patients were first seen at the start of their reactions. All were classified clinically and histologically according to the Ridley-Jopling scale ${ }^{3} ; 16$ were borderline tuberculoid, two borderline, and six borderline lepromatous. Ten of the patients were male and 14 female, and their ages ranged from 12 to 41 years.

All of the patients were on dapsone, which was continued in unaltered dosage throughout the study. Reactions were treated with prednisolone. The initial dosage was $30-40 \mathrm{mg}$ daily, which was gradually reduced over one to nine months depending on the duration and severity of the reaction.

\section{ASSESSMENTS DURING REACTION}

The following assessments were made repeatedly during the reactions and in the post-reaction periods, which varied from one to nine months.

Skin disease was rated arbitrarily as 0 to $3+$ according to the degree of erythema and oedema of skin lesions.

Nerve disease was assessed by three criteria, at least two of which had to be satisfied for nerve involvement to be considered to be marked-(a) clinical assessment of nerve tenderness and swelling, recorded as absent $(0)$, slight $( \pm)$, or marked $(+) ;(b)$ motor nerve conduction studies of the ulnar and median nerves (below $55 \mathrm{~m} / \mathrm{s}$ was regarded as abnormal); (c) voluntary muscle tests ${ }^{4}$ (sustained fall in muscle power in two or more muscle groups supplied by nerve indicated deterioration $(t)$; lesser changes $(0)$ were disregarded).

$L T$ tests were performed at least twice during the reactions, whole and "sonicated" preparations of $M$ leprae being used as antigens, and phytohaemagglutinin being used to show cell viability.

Whenever the LT tests were performed dose-response curves were drawn for each antigen and the maximum values for the two preparations of $M$ leprae added together. The concentrations with the highest total, representing the peak of the reaction, are those reported here. Similarly, assessments of skin and nerve disease are the maximum figures recorded.

\section{PREPARATION OF ANTIGENS}

The same antigen preparations were used in all tests and were derived from a large subcutaneous nodule excised from a patient with lepromatous leprosy. The nodule was homogenised and the bacilli were washed in phosphate-buffered saline at $\mathrm{pH} 7 \cdot 2$. The suspension was stored at $-20^{\circ} \mathrm{C}$. This preparation is referred to as whole $M$ leprae. The bacilli in part of the same suspension were exposed to sonic vibration until no acid-fast bacilli remained. This preparation is referred to as sonicated, and was also stored at $-20^{\circ} \mathrm{C}$.

\section{TECHNIQUE OF LT TEST}

In all tests both $M$ leprae antigen preparations were used at three concentrations standardised to the bacillary content of the original homogenate-that is, $10^{6}, 10^{7}$, and $10^{8}$ bacilli $/ \mathrm{ml}$. A micromethod was used. Peripheral blood lymphocytes were separated on Ficoll Isopaque, washed three times, and cultured in tissue culture medium 199 (Gibco) at a concentration of $10^{6} / \mathrm{ml}$. Triplicate cultures were 
incubated with the appropriate antigen preparation in $5 \% \mathrm{CO}_{2}$ in air for seven days at $37^{\circ} \mathrm{C}$ and $100 \%$ humidity. Control cultures without antigen added were also included in the tray. Sixteen hours before harvesting, $0.5 \mu \mathrm{Ci}$ tritiated thymidine $\left({ }^{3} \mathrm{H}-\mathrm{TdR}\right.$; specific activity $2 \mathrm{Ci} / \mathrm{mmol}$ ) was added to each well. The contents of the wells were harvested on to glass-fibre filters and the ${ }^{3} \mathrm{H}-\mathrm{TdR}$ uptake was recorded as counts/min using a liquid scintillation counter.

\section{Results}

The LT responses (with control values subtracted) in the 15 patients studied from the inception of antileprosy treatment at baseline (before treatment), during a reaction, and in the post-reaction period are shown in the fig. Thirteen of the patients showed an appreciable increase in response with the two antigen preparations during a reaction, with a comparable fall in the post-reaction period. Both the rise and fall were highly significant $(P<0.005$; Student's $t$ test).

The rise in LT response was due, in different patients, to increased responses to whole or sonicated preparations of $M$ leprae or both. When the total LT responses in these patients when in reaction, and in the nine further patients who were also in reaction though they had not been followed from the inception of treatment, were studied the clinical presentation was found to correlate with the rise in LT with the two different antigen preparations. In the 10 patients judged to have a skin reaction clinically there was an appreciable increase in LT when whole $M$ leprae was used but a smaller or negligible increase when sonicated $M$ leprae was used. In those judged to have a nerve reaction the reverse was true. Those with mixed reactions showed a high LT response with both antigen preparations. The table gives a summary of these results, together with the LT values expressed as a ratio of whole to sonicated $M$ leprae (total responses). The mean ratios for the patients with skin, mixed, and nerve reactions were $2.91,0.94$, and 0.50 respectively. Statistical significance was estimated with the nonparametric test of Kruskal and Wallis ${ }^{5}$ for independent samples, the differences between the three groups being highly significant $(\mathbf{P}<0.001)$.

\section{Discussion}

The prospective study on the 15 borderline leprosy patients showed an appreciable increase in LT responses to antigens of $M$ leprae during reversal reactions, and confirmed that the mechanism of these reactions is an increase in cell-mediated immunological reactivity. The results of LT tests in all 24 patients in reaction showed that nerve involvement and skin disease are associated with responses to different antigens. The

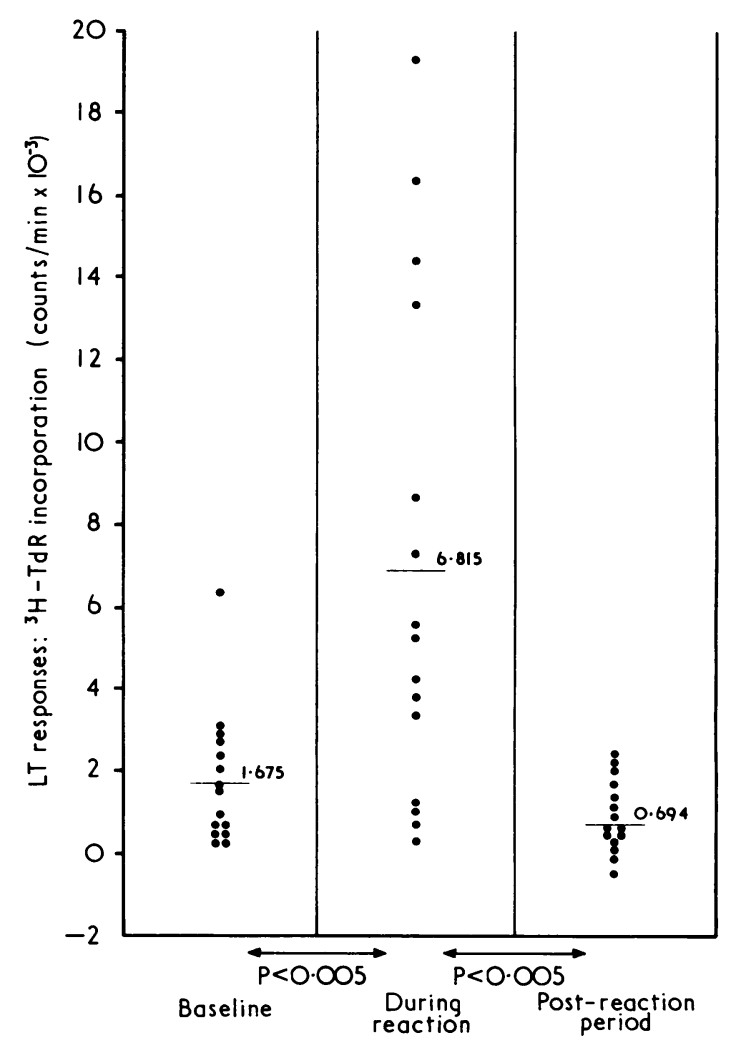

LT responses in 15 patients before (baseline), during, and after reaction. Responses to whole and sonicated $M$ leprae are added, with control values subtracted.

failure of whole washed bacilli to elicit a high response in nerve reactions suggests that cytoplasmic antigens play an important part in this condition. In skin reactions the increased response is chiefly directed at whole washed bacilli, indicating that surface antigens are more important. The smaller response to sonicated preparations (in which surface antigens are present) may be a dilution affect.

The mechanism of nerve reactions may therefore be as follows. In some borderline tuberculoid leprosy patients immunologically competent enough to recognise and destroy the leprosy bacillus many bacilli may be present in the nerve but few if any may be

Clinical manifestations related to $L T$ responses in 24 patients in reaction

\begin{tabular}{|c|c|c|c|c|c|c|c|c|c|}
\hline \multirow{3}{*}{$\begin{array}{l}\text { Case } \\
\text { No }\end{array}$} & \multirow{3}{*}{$\begin{array}{c}\text { Biopsy } \\
\text { classification* }\end{array}$} & \multicolumn{5}{|c|}{ Clinical assessment } & \multicolumn{2}{|c|}{ LT (counts min) } & \multirow{3}{*}{$\begin{array}{l}\text { Ratio of whole } \\
\text { to sonicated } \\
\text { M leprue }\end{array}$} \\
\hline & & \multirow{2}{*}{ Skin } & \multicolumn{3}{|c|}{ Nerve $†$} & \multirow{2}{*}{ Reaction } & \multirow{2}{*}{$\begin{array}{c}\text { Whole } \\
\text { M leprae }\end{array}$} & \multirow{2}{*}{$\begin{array}{l}\text { Sonicated } \\
M \text { leprae }\end{array}$} & \\
\hline & & & C & $\mathbf{N}$ & M & & & & \\
\hline $\begin{array}{r}1 \\
2 \\
3 \\
4 \\
5 \\
6 \\
7 \\
8 \\
9 \\
10\end{array}$ & $\begin{array}{l}\text { BT } \\
\text { BT } \\
\text { BT } \\
\text { BT } \\
\text { BB } \\
\text { BT } \\
\text { BT } \\
\text { BL } \\
\text { BB } \\
\text { BL }\end{array}$ & $\begin{array}{l}2+ \\
2+ \\
3+ \\
2+ \\
3+ \\
2+ \\
2+ \\
2+ \\
2+ \\
2+\end{array}$ & $\begin{array}{l}0 \\
0 \\
\pm \\
. \\
0 \\
0 \\
. \\
0 \\
+ \\
+ \\
+\end{array}$ & $\begin{array}{c}\text { ND } \\
0 \\
0 \\
0 \\
0 \\
0 \\
0 \\
0 \\
0 \\
0\end{array}$ & $\begin{array}{c}0 \\
0 \\
0 \\
0 \\
\text { ND } \\
0 \\
0 \\
0 \\
0 \\
0\end{array}$ & $\begin{array}{l}\text { Skin } \\
\text { Skin } \\
\text { Skin } \\
\text { Skin } \\
\text { Skin } \\
\text { Skin } \\
\text { Skin } \\
\text { Skin } \\
\text { Skin } \\
\text { Skin }\end{array}$ & $\begin{array}{r}13861 \\
11799 \\
4306 \\
2665 \\
6493 \\
7022 \\
7195 \\
4196 \\
7396 \\
2925\end{array}$ & $\begin{array}{r}1770 \\
2353 \\
950 \\
1029 \\
3462 \\
3751 \\
4434 \\
3014 \\
6123 \\
2484\end{array}$ & $\begin{array}{l}7.83 \\
5.01 \\
4.53 \\
2.59 \\
1.88 \\
1.87 \\
1.62 \\
1.39 \\
1.21 \\
1.18\end{array}$ \\
\hline $\begin{array}{l}11 \\
12 \\
13 \\
14 \\
15 \\
16\end{array}$ & $\begin{array}{l}\text { BT } \\
\text { BT } \\
\text { BT } \\
\text { BT } \\
\text { BT } \\
\text { BT }\end{array}$ & $\begin{array}{l}2+ \\
2+ \\
+ \\
2+ \\
2+ \\
2+\end{array}$ & $\begin{array}{l}+ \\
+ \\
+ \\
+ \\
+ \\
+\end{array}$ & $\begin{array}{l}+ \\
+ \\
+ \\
\text { ND } \\
+ \\
+\end{array}$ & $\begin{array}{l}+ \\
+ \\
+ \\
+ \\
+ \\
0\end{array}$ & $\begin{array}{l}\text { Mixed } \\
\text { Mixed } \\
\text { Mixed } \\
\text { Mixed } \\
\text { Mixed } \\
\text { Mixed }\end{array}$ & $\begin{array}{r}12089 \\
3450 \\
1550 \\
9050 \\
3763 \\
1086\end{array}$ & $\begin{array}{r}10031 \\
3174 \\
1658 \\
9950 \\
5032 \\
1494\end{array}$ & $\begin{array}{l}1.21 \\
1.09 \\
0.93 \\
0.91 \\
0.75 \\
0.73\end{array}$ \\
\hline $\begin{array}{l}17 \\
18 \\
19 \\
20 \\
21 \\
22 \\
23 \\
24\end{array}$ & $\begin{array}{l}\text { BT } \\
\text { BL } \\
\text { BT } \\
\text { BL } \\
\text { BT } \\
\text { BL } \\
\text { BT } \\
\text { BL }\end{array}$ & $\begin{array}{l}0 \\
0 \\
0 \\
0 \\
0 \\
0 \\
0 \\
0\end{array}$ & $\begin{array}{l}+ \\
+ \\
+ \\
+ \\
+ \\
+ \\
+ \\
+\end{array}$ & $\begin{array}{c}+ \\
+ \\
\text { ND } \\
\text { ND } \\
+ \\
+ \\
+ \\
\text { ND }\end{array}$ & $\begin{array}{l}+ \\
+ \\
+ \\
+ \\
+ \\
+ \\
+ \\
+\end{array}$ & $\begin{array}{l}\text { Nerve } \\
\text { Nerve } \\
\text { Nerve } \\
\text { Nerve } \\
\text { Nerve } \\
\text { Nerve } \\
\text { Nerve } \\
\text { Nerve }\end{array}$ & $\begin{array}{r}359 \\
5187 \\
1232 \\
827 \\
1626 \\
2156 \\
1086 \\
261\end{array}$ & $\begin{array}{r}357 \\
6669 \\
1967 \\
1831 \\
4749 \\
6873 \\
3693 \\
1136\end{array}$ & $\begin{array}{l}1.01 \\
0.78 \\
0.63 \\
0.45 \\
0.34 \\
0.31 \\
0.29 \\
0.23\end{array}$ \\
\hline
\end{tabular}

${ }^{*}$ Ridley-Jopling scale. ${ }^{3} \quad \mathrm{BT}=$ Borderline tuberculoid. $\quad \mathrm{BB}=$ Borderline. $\quad \mathrm{BL}=$ Borderline lepromatous

$+\mathrm{C}=$ Clinical assessment. $\mathrm{N}=$ Motor nerve conduction velocity studies. $\quad \mathrm{ML}=$ Voluntary muscle testing. $\mathrm{ND}=\mathrm{Not}$ done. 
present in the skin. ${ }^{6}$ The same phenomenon has been noted in borderline lepromatous patients, particularly afer one or two years of treatment. This is presumably because Schwann cells, in which most of the bacilli are contained, are relatively long-lived. In this situation probably the surface antigens of the bacillus are rarely exposed; soluble cytoplasmic antigens are more likely to be exposed but they are probably released slowly, so that few lymphocytes are sensitised. In reactions there appears to be a sudden increase in the release of these cytoplasmic antigens, though the trigger mechanism is unknown. Subsequent destruction of the Schwann cells may then lead to increased exposure of bacillary surface antigens, and it was noted that in five of the eight patients with nerve reactions there was a rise in LT responses when whole $M$ leprae was used as antigen in the weeks after the reaction began.

In skin reactions the $L T$ results indicate that there may be an increased exposure of bacillary surface antigens. In borderline tuberculoid leprosy few bacilli are present in the skin and they are predominantly found in the dermal nerves. It appears that the lymphocytes and macrophages in the skin suddenly recognise the bacilli in the dermal nerves, with destruction of the Schwann cells and consequent exposure of surface antigens. This possibility is borne out by the observation that in eight out of 10 of our patients with skin or mixed reactions who had skin biopsies during reaction large nerves were present containing degenerate bacilli within granuloma.

In mixed reactions hypersensitivity reaction in nerves often occur weeks after the reaction in skin. This may be because some cytoplasmic antigens are released during the "skin phase" with increased lymphocyte sensitisation to these antigens, leading to increased recognition of such antigens elsewhere-particularly those in nerves.
Although the trigger mechanism for these reactions remains unknown, it has been suggested that coincidental exposure to other mycobacteria ${ }^{1}$ may be a cause. There is no good evidence for this, however, and in those of our patients on whom LT tests were performed using BCG and $M$ duvalii as antigens during reactions there was no consistent concomitant rise in responses.

Reversal reactions are of great importance as they may lead to nerve destruction almost overnight. Unfortunately this usually occurs when the patient is receiving antileprosy treatment, the more so if he has been diagnosed at an early stage of the disease, before nerve damage has occurred. It is important to elucidate the precipitating factors that cause these reactions, and when this is achieved it may be possible to prevent them.

We thank Monica Lovgren and Kesete Negassi for technical help, Dr B Naafs for nerve conduction studies, and Dr D S Ridley for independent histological classification of the patients. We also thank the staff of the All Africa Leprosy Rehabilitation and Training Centre for referral of patients to us and provision of clinical facilities.

\author{
References \\ ${ }^{1}$ Godal, T, et al, Acta Pathologica et Microbiologica Scandinavica, 1973, \\ section A, suppl No 236, p 45. \\ ${ }^{2}$ Wemambu, S N C, et al, Lancet, 1969, 2, 933. \\ ${ }^{3}$ Ridley, D S, and Jopling, W H, International fournal of Leprosy, 1966, 34, \\ 255. \\ 4 Goodwin, C S, Leprosy Review, 1968, 39, 209. \\ ${ }^{5}$ Kruskal, W H, Annals of Mathematical Statistics, 1952, 23, 525. \\ ${ }^{6}$ Weddell, A G M, and Pearson, J M H, in Topics on Tropical Neurology, \\ ed R W Hornabrook. Philadelphia, Davis, 1975.
}

\section{Summary}

Thyroid function and serum levels of triiodothyronine (T3) and thyroxine (T4) were investigated in 79 euthyroid geriatric patients. Of the 59 inpatients and 20 outpatients $35(59 \%)$ and 2 , respectively, had low T3 levels. In contrast, $7(12 \%)$ and $6(30 \%)$, respectively, had raised T4 levels. Two further patients were excluded from the study because of raised levels of thyroid-stimulating hormone. Thyroxine-binding globulin was greatly increased in both groups of patients, but low serum albumin levels were present in $31\left(39^{\circ}{ }_{0}\right)$. Despite these changes free T3 and T4 indices closely followed total T3 and T4

\footnotetext{
Department of Endocrinology, Radcliffe Infirmary, Oxford OX2 6HE

A W BURROWS, MRCP, registrar in medicine

$R$ A SHAKESPEAR, BA, research assistant

E COOPER, B SC, technician

C M AICKIN, B SC, technician

C W BURKE, DM, FRCP, consultant physician

Department of Clinical Endocrinology, Medizinische Hochschule Hanover, Germany

R D HESCH, MD, privatdozent
}

levels. The difference between the two groups of patients did not correlate with body weight, diagnostic categories, age, drug treatment, or duration of stay in hospital.

\section{Introduction}

Low blood levels of triiodothyronine (T3) are common in debilitating diseases. ${ }^{1}$ In view of the reported prevalence of thyroid disease in the elderly ${ }^{2-4}$ (which is disputed by some workers ${ }^{5}{ }^{6}$ ) we investigated thyroid function and serum levels of $\mathrm{T} 3$ and thyroxine (T4) in patients in our geriatric hospital.

\section{Patients and methods}

Sera were obtained from 64 inpatients (mean age 79.6 years, range 69-95 years) and 24 outpatients (mean age $81 \cdot 8$ years, range $65-93$ years). About a third of the inpatients were chronically disabled or ill to a degree preventing their return home, though most were eventually discharged, and a third were convalescent or being rehabilitated after acute episodes. These patients were studied between two and 30 weeks after admission. The remaining inpatients were studied within one week of admission, having suffered an acute episode or gradual general deterioration sufficient to need admission. There was no difference in our findings between the three groups. All had multiple disorders, usually referrable to the cardiovascular or nervous system. Nine $(14 \%)$ died within six weeks of our studies.

The outpatients were attending for follow-up after discharge or for maintenance of contact with social or rehabilitation services. Their general medical condition differed from that of the longer-stay 